\title{
Evidencias de validez de constructo y de criterio de la Escala Massie-Campbell de Apego Durante Estrés (ADS)
}

\author{
MAGALY NÓBLEGA* \\ Pontificia Universidad Católica del Perú \\ ORCID: https://orcid.org/0000-0001-6572-813X \\ GABRIELA CONDE \\ Pontificia Universidad Católica del Perú \\ ORCID: https://orcid.org/0000-0002-6103-1025 \\ JUAN NUÑEZ DEL PRADO \\ Pontificia Universidad Católica del Perú \\ ORCID: https://orcid.org/://0000-0001-5502-514X \\ PATRICIA BÁRRIG \\ Pontificia Universidad Católica del Perú \\ ORCID: https://orcid.org/0000-0003-4685-5760 \\ FRANCESCO MARINELLI \\ Pontificia Universidad Católica del Perú \\ ORCID: https://orcid.org/0000-0002-0470-0130 \\ NATALI ALCÁNTARA \\ Pontificia Universidad Católica del Perú \\ ORCID: https://orcid.org/0000-0003-4721-7023 \\ RODRIGO CÁRCAMO \\ Universidad de Magallanes, Chile \\ ORCID: https://orcid.org/0000-0002-9227-7132
}

How to quote this article: Nóblega, M., Conde, G., Nuñez del Prado, J., Bárrig, P., Marinelli, F., Alcántara, N. \& Cárcamo, R. A. (2019). Evidencias de validez de constructo y de criterio de la Escala Massie-Campbell de Apego Durante Estrés (ADS). Acta Colombiana de Psicología, 22(1), 129-140. doi: http://www.dx.doi.org/10.14718/ ACP.2019.22.1.7

\section{Resumen}

\begin{abstract}
La escala Massie-Campbell de Apego Durante Estrés (ADS; 1983), es una guía de observación de la interacción entre madres (o cuidadores) y sus hijos de 6 a 18 meses. Este estudio busca obtener evidencias de validez de constructo (convergente y divergente) y de criterio (sensibilidad, especificidad y valores predictivos) de dicho instrumento. Para ello, se evaluó el apego en 32 niños y niñas de ocho a diez meses de Lima, Perú, utilizando el ADS y el Attachment Q-set 3.0 (AQS), de Waters (1995), así como la sensibilidad de sus madres. Como resultado, se encontró que las clasificaciones del apego seguro e inseguro dadas a partir del ADS se encuentran relacionadas con los puntajes continuos de seguridad del apego del $\mathrm{AQS}(r=.41, p=.02)$ y con las clasificaciones dicotómicas (seguro vs. inseguro) obtenidas a partir del mismo $\left[\chi^{2}(1, N\right.$ $=32)=4.69, p=.03, d=.83]$. Adicionalmente, no se hallaron diferencias significativas en la sensibilidad materna de las madres de los niños clasificados como seguros y la de los inseguros. Dado que la sensibilidad de la escala ADS no alcanzó niveles satisfactorios $(33.3 \%$, IC $95 \%=[15.48 ; 56.90])$, de manera exploratoria se evaluó un punto de corte de cinco conductas seguras, el cual mejora su sensibilidad $(47.6 \%$, IC $95 \%=[26.4 ; 69.7])$ y mantiene sus niveles satisfactorios de especificidad $(90.9 \%$, IC $95 \%=[57.1 ; 99.5])$. En conclusión, el instrumento muestra adecuadas evidencias de validez de constructo convergente, pero no divergente; y, al ser considerado un instrumento de tamizaje, su sensibilidad, especificidad y valores predictivos necesitan seguir siendo estudiados con el fin de conseguir un punto de corte con mayor validez.

Palabras clave: apego, escala Massie-Campbell, ADS, validez.
\end{abstract}

\footnotetext{
* Docente principal del Departamento de Psicología, Av. Universitaria 1801, San Miguel, Lima, Perú. Teléfono: 6262000 , anexo 4534. mnoblega@pucp.pe
} 


\title{
Construct and criterion validity evidences of the Massie-Campbell Scale of Attachment During Stress (ADS)
}

\begin{abstract}
The Massie-Campbell Attachment During Stress Scale (ADS, Massie \& Campbell, 1983) is an observation guide of the interactions between mothers (or caretakers) and children from 6 to 18 months. This study sought the construct validity (convergent and discriminant) of ADS, as well as its criterion validity (sensitivity, specificity, and predictive values). To that end, child attachment was measured in a group of 32 children aged 8 to 10 months from Lima, Peru using ADS and Attachment Q-set 3.0 (AQS, Waters, 1995). Maternal sensitivity was assessed as well. It was found that ADS attachment classifications were related with AQS attachment security continuous scores $(r=.41, p=.02)$ and with its dichotomous classifications [secure vs. insecure; $\chi^{2}(1, N=32)=4.69, p=0.03, d=0.83$ ]. Additionally, no significant differences between the ADS's maternal sensitivity of mothers with children classified as secure and mothers with children classified as insecure were found. Since ADS's sensitivity did not reach satisfactory levels $(33.3 \%, 95 \% \mathrm{CI}=[15.48,56.90])$, a cut-off point of 5 secure behaviors was used, which improved its sensitivity $(47.6 \%, 95 \% \mathrm{CI}=[26.4 ; 69.7])$ while maintaining satisfactory levels of specificity $(90.9 \%, 95 \% \mathrm{CI}=[57.1 ; 99.5])$. The instrument shows adequate convergent validity but lacks evidence of discriminant validity. Further exploration of ADS's sensitivity, specificity, and predictive values is recommended in order to obtain a cut-off point with greater validity, given that the instrument is considered a screening test.

Keywords: attachment, Massie-Campbell Scale, ADS, validity.
\end{abstract}

\section{Evidências de validade de constructo e de critério da Escala Massie- Campbell de Apego durante o Stress (ADS)}

Resumo

\begin{abstract}
A Escala Massie-Campbell de Apego durante o Stress (ADS; 1983) é um guia de observação da interação entre mães (ou cuidadores) e seus filhos de 6 a 18 meses. Este estudo procura obter evidências de validade de constructo (convergente e divergente) e de critério (sensibilidade, especificidade e valores preditivos) desse instrumento. Para isso, foi avaliado o apego em 32 crianças de oito a dez meses, de Lima, Peru, utilizando o ADS e o Attachment Q-set 3.0 (AQS), de Waters (1995), bem como a sensibilidade de suas mães. Como resultados, constatou-se que as classificações do apego seguro e inseguro dadas a partir do ADS se encontram relacionadas com as pontuações contínuas de segurança do apego do AQS $(r=.41, p=.02)$ e com as classificações dicotômicas (seguro versus inseguro) obtidas a partir deste $\left[\chi^{2}(1, N=32)=4.69, p=.03, d=.83\right]$. Além disso, não foram encontradas diferenças significativas na sensibilidade materna das mães das crianças classificadas como seguras e a das inseguras. Tendo em vista que a sensibilidade da escala ADS não atingiu níveis satisfatórios (33.3 \%, IC $95 \%=[15.48 ; 56.90])$, de maneira exploratória, foi avaliado um ponto de corte de cinco comportamentos seguros, o que melhora sua sensibilidade $(47.6 \%$, IC $95 \%=[26.4 ; 69.7])$ e mantém seus níveis satisfatórios de especificidade (90.9 \%, IC $95 \%=[57.1 ; 99.5])$. Em conclusão, o instrumento mostra adequadas evidências de validade de constructo convergente, mas não divergente; e, ao ser considerado um instrumento de rastreamento, sua sensibilidade, especificidade e valores preditivos precisam continuar sendo estudados a fim de conseguir um ponto de corte com maior validade.

Palavras-chave: apego, escala Massie-Campbell, ADS, validade.
\end{abstract}

\section{Introducción}

La teoría del apego desarrollada por Bowlby (1969) define el apego como el vínculo afectivo que une al niño con su cuidador principal a través del tiempo y del espacio (Ainsworth, Blehar, Waters \& Wall, 1978). Según esta teoría, los seres humanos tienen la necesidad innata de desarrollar el apego con una o varias personas que le proporcionen cuidado y protección. Por esta razón, nacen con una serie de conductas instintivas denominadas conductas de apego -llanto, orientación de la mirada, vocalización, aprehensión, entre otras-, que tienen el objetivo de propiciar la proximidad de su figura de cuidado. Estas conductas de apego, con base en el desarrollo mental y físico del infante, así como en las repetidas experiencias de interacción con su cuidador, se organizan en estrategias especializadas para lograr la proximidad física o afectiva con la figura de apego, mientras que, de forma paralela, desarrolla conductas de exploración dirigidas a conocer y manejar el entorno físico y social; conductas que le permitirán alejarse paulatinamente 
de su figura de apego y dirigir su atención hacia estímulos del contexto.

El equilibro entre las conductas de búsqueda de proximidad y de exploración se denomina conducta de base segura, y es entendida como la capacidad del niño para usar a su cuidador principal como una base segura con el fin de explorar su entorno y regresar en momentos de amenaza o estrés (Bowlby, 1988). A partir de esta definición, el logro de dicho equilibrio denotaría la existencia de un vínculo de apego de tipo seguro, mientras que el desbalance hacia uno de los dos extremos configuraría un apego inseguro -específicamente, un apego inseguro evitativo en caso de un desbalance hacia la exploración, y un apego inseguro ambivalente para el desequilibro hacia el extremo de la búsqueda de proximidad-(Cassidy, 2016).

Con respecto a la evaluación de la conducta de base segura, el instrumento más importante para evaluarla es el Strange Situation Procedure (SSP; Ainsworth et al., 1978), o Procedimiento de la Situación Extraña, ya que, en este, se clasifica la conducta del niño en alguno de los tipos de apego a partir de su observación en una situación de laboratorio generadora de un estrés moderado. Sin embargo, a pesar de ser considerado el gold standard, algunos autores han identificado limitaciones relacionadas con sus procedimientos de aplicación, con su sistema de calificación y con su validez ecológica (Clarke-Stewart, Goosens \& Allhusen, 2001; Waters \& Deane, 1985). Así, por ejemplo, se considera que el rango de edad de aplicación es muy corto, que el tiempo recomendado entre aplicaciones es muy largo, y que tiene altos costos de administración y calificación. Además, sobre su sistema de calificación se afirma que los resultados taxonómicos proporcionan información limitada sobre el sistema de apego del niño y limitan las opciones de análisis; y sobre su validez ecológica se considera que la ausencia de la madre como fuente de estrés podría interferir en el despliegue regular de las conductas de apego de los niños de determinadas culturas que pueden estar más familiarizados con la rutina de quedarse con un extraño.

En respuesta a algunas de estas limitaciones surge el Attachment Q-set 3.0 (AQS; Waters, 1995), considerado el segundo instrumento más importante para la evaluación del apego (van IJzendoorn, Vereijken, Bakermans-Kranenburg \& Riksen-Walraven, 2004), cuya finalidad es evaluar la organización de la conducta de base segura utilizando la metodología Q-sort. Específicamente, este procedimiento requiere de la observación de la interacción cuidador-niño durante un periodo de dos a seis horas, y brinda como resultado el nivel de seguridad del apego del niño al correlacionar la conducta observada con el criterio de seguridad previamente definido por expertos.
Este instrumento es adecuado para su uso, lo cual se ve reflejado en los buenos indicadores de la evaluación del apego en infantes y preescolares en diversos contextos. Así, Cadman, Diamond y Fearon (2017), en un meta-análisis que incorporó 268 muestras independientes, reportaron adecuadas evidencias de validez del instrumento al encontrar un tamaño de efecto moderado $(r=.20)$ entre los puntajes del AQS (utilizado por un observador) y las clasificaciones dadas por el SSP $(r=.39$ para observaciones de más de $180 \mathrm{~min})$, así como una asociación significativa $(r=.17)$ con el apego desorganizado en comparación con las otras categorías del SSP combinadas. También, encontraron una relación entre los puntajes AQS (observado) con la sensibilidad del cuidador -con un buen tamaño del efecto $(r=.32)-$, y con la competencia socioemocional del niño $(r=.24)$; al igual que una asociación significativa, $\mathrm{y}$ en el sentido esperado, con el temperamento del niño $(r=.27)$. Asimismo, encontraron adecuados niveles de confiabilidad para este instrumento, ya que, a partir del análisis de 12 estudios, encontraron asociaciones significativas a través del tiempo para los puntajes observados en las evaluaciones test-retest $(r=.41)$, así como un acuerdo entre los puntajes auto-reportados y los observados $(r=.43)$.

Por otra parte, el AQS ha sido utilizado en el Perú en diferentes estratos socioeconómicos tanto en ambientes familiares como no familiares y ha mostrado adecuadas características psicométricas (Conde, Nóblega, Fourment \& Nuñez del Prado, 2015; Nóblega, Fourment, Núñez del Prado, Conde \& Bahamonde, 2015; Posada et al., 2013; Posada et al., 2016), con variaciones entre .43 y .60 para los puntajes de seguridad del AQS y los de la sensibilidad materna-medidos con el Maternal Behavior for Preschoolers Q-set-; con índices de confiabilidad inter-evaluador entre .76 y .96 (Buitrón, 2011; Conde et al., 2015; Dávila, 2013; Nóblega, 2012; Posada et al., 2013; Posada et al., 2016; Ugarte, 2014); y con su utilización de forma exploratoria para evaluar los precursores de la conducta de base segura en niños menores de un año (Nóblega et al., 2016).

Sin embargo, al igual que el SSP, el AQS cuenta con algunas limitaciones, tales como un periodo de observación muy largo y un sistema de calificación complejo, que, dependiendo del nivel de experiencia del codificador, puede tardar entre 30 y 60 minutos; características que dificultan su uso para investigaciones de gran escala.

Frente a ello, en los últimos años un instrumento alternativo ha tomado relevancia en el ámbito latinoamericano, conocido como Escala de Apego Durante Estrés (ADS; Massie-Campbell, 1983), que permite identificar los patrones de interacción potencialmente problemáticos (del tipo evitativo o ambivalente), así como la falta de sincronía en la interacción madre-niño; y cuenta con adecuadas 
132

propiedades psicométricas para la evaluación de la calidad de la interacción de las diadas niño-cuidador en situaciones de estrés (Massie \& Campbell, 1983, 1992; Tryphonopoulos, Letourneau \& Ditommaso, 2014).

Además de esto, dada la gran importancia del comportamiento del cuidador para el despliegue de las conductas de apego del niño, la calificación de la ADS toma en cuenta tanto las conductas del niño como las del cuidador en dos subescalas separadas (Massie \& Campbell, 1983). Algunas de sus ventajas frente a otros instrumentos son su aplicación a diadas con niños de edades tempranas (desde el nacimiento hasta los 18 meses), su corto periodo de observación (entre cinco y quince minutos) y que puede ser utilizada para la observación tanto en el ámbito natural como en situaciones estructuradas o de laboratorio (Massie \& Campbell, 1992). No obstante, si bien la ADS ha sido utilizada en el ámbito de la investigación (Figueroa, Binda \& Santelices, 2012; Kotliarenco, Gómez, Muñoz \& Armijo, 2009; Lecannelier et al., 2009; Leigh, Vergara \& Santelices, 2012), la evidencia de sus características psicométricas es escasa (UIT, s. f.), y son pocos los estudios que se han dedicado exclusivamente a esto.

La investigación de Cárcamo, van IJzendoorn, Vemeer y van der Veer (2014) es el estudio psicométrico más reciente que da cuenta de diversas evidencias de validez de la ADS, donde, con respecto a su validez convergente, se encontró una asociación significativa entre las clasificaciones de seguro y no seguro de la escala total ADS general y la SSP $\left[\chi_{(1, N=69)}^{2}=13.20, p<.001, d=0.96\right]$ y para la sub-escala de la conducta del niño $\left[\chi_{(1, N=69)}^{2}=4.94, p=.026, d=0.50\right]$. No obstante, al realizar la comparación para los tres tipos de apego se encontró que, aunque las clasificaciones de ambas escalas estaban asociadas, había una sobre-representación del apego seguro y una sub-representación del tipo de apego evitativo por parte de la ADS. Por otra parte, con respecto a las evidencias de validez divergente se encontró que el grupo clasificado como inseguro con la ADS tenía madres que puntuaban significativamente más bajo en sensibilidad materna que el grupo clasificado como seguro $\left(M_{\text {madres de }}\right.$ niños inseguros $=4.70 ; D E_{\text {madres de niños inseguros }}=1.35 ; M_{\text {madres de niños }}$ $\underset{\text { seguros }}{\text { niños inseguros }}=5.66 ; D E \underset{\text { madres de niños seguros }}{\text { m }}=1.14, t=-2.85, g l=67$, $p=.006, d=0.76)$. Y, por último, con respecto a su validez de contenido, a partir de la solicitud a un grupo de cuatro expertos en apego para que clasificara los enunciados de la ADS acorde a los tres tipos de apego que contempla la escala, 40 de los 70 enunciados fueron clasificados como correctos por los cuatro expertos.

A partir de estos resultados, Cárcamo et al., 2014 concluyeron que la ADS tiene un desempeño "satisfactorio en la distinción entre los niños seguros y los niños inseguros" (p. 772) tanto al tener en cuenta la puntuación del niño y de la madre en conjunto, como considerando solo las puntuaciones del niño; pero que, a un nivel más detallado, tiene dificultades para discriminar entre los diversos tipos de apego, por lo que recomendaron que los resultados fueran interpretados con cautela. Además, considerando las limitaciones de su estudio, recalcaron la importancia de la evaluación de las características psicométricas de dicho instrumento en un contexto natural (no de laboratorio) y con muestras de características sociales y económicas distintas a las de su estudio.

De esta manera, y tomando en cuenta que el apego ha sido priorizado como un resultado importante del desarrollo infantil temprano en la política pública de diversos países latinoamericanos -entre los que se encuentra el Perú-, resulta relevante contar con un instrumento de evaluación corto y de fácil aplicación con evidencias de validez que faciliten su uso a gran escala. Por ello, el presente estudio de tipo psicométrico o instrumental tuvo el objetivo de analizar las evidencias de validez de constructo (convergente y divergente) y de criterio (análisis de la sensibilidad, especificidad y valores predictivos) de la escala ADS en un grupo de madres y sus niños de ocho a diez meses de Lima, Perú ${ }^{1}$.

Para generar evidencias de validez de constructo convergente se compararon los puntajes de la ADS con los del AQS, instrumento escogido dado su funcionamiento con niños menores de un año probado en el contexto peruano (Nóblega et al., 2016). Para las evidencias de validez de constructo divergente -considerando que existen estudios que identifican a la sensibilidad materna como un constructo diferente, pero asociado al desarrollo de un apego seguro (Nóblega, 2012; Nóblega et al., 2016; Posada et al., 2013; Posada et al., 2016; Jin, Jacobvitz, Hazen \& Jung, 2012; Van IJzendoorn, Bakermans-Kranenburg \& Sagi-Schwartz, 2006)-, se evaluó la relación entre los puntajes de la ADS y los puntajes de sensibilidad de las madres evaluados con el MBQS 2.1 (Pederson \& Moran, 1995; Pederson, Moran \& Bento, 1999). La sensibilidad, la especificidad y los valores predictivos de la ADS se calcularon utilizando como criterio los puntajes dicotómicos del AQS.

\section{Método}

\section{Participantes}

Participaron 32 madres, con sus hijos, de la ciudad de Lima, Perú. Su media de edad era de 29.6 años $(D E=6.28$,

\footnotetext{
1 Para la clasificación de evidencias de validez se utilizó como referencia el "modelo para la descripción y evaluación de pruebas psicológicas y educativas revisado" de la European Federation of Psychologist's Association - EFPA (Evers et al., 2013).
} 
Min $=19$, Max $=44)$, el $43.7 \%$ había culminado el nivel educativo de educación superior técnica o universitaria, el $53.1 \%$, educación secundaria y el $3.1 \%$, la educación primaria; el $84.4 \%$ era casada o conviviente, y el $81 \%$ se dedicaba principalmente al trabajo doméstico en su hogar. Los niños y niñas participantes fueron 20 niños $(62.50 \%)$ y 12 niñas $(37.5 \%)$ con un rango de edad entre los ocho y los diez meses y 29 días de edad $(M=8.91 ; D E=0.96)$. En todos los casos la madre era el cuidador principal; y, a partir del reporte materno, se verificó que ninguno de los niños presentara una evidente patología grave del desarrollo o de salud (retardo mental, autismo, síndrome de Down o similares, o prematuridad).

\section{Consideraciones éticas}

Este estudio contó con la aprobación del Comité de Ética de la universidad financiadora. La selección de las diadas participantes se realizó por conveniencia, ya que se eligió a algunas de las madres que llevaban a sus hijos a un centro de salud del Estado. A cada madre se le explicó el estudio y se le invitó a participar del mismo -solo en un caso la madre fue referida por un contacto de uno de los investigadores del equipo, y esta era residente de la zona de estudio-; y las madres que aceptaron participar voluntariamente firmaron el consentimiento informado, en el cual se describía el procedimiento a seguir, con los respectivos beneficios y posibles riesgos, y se aseguraba el carácter voluntario de su participación, la posibilidad de retirarse en cualquier momento, $\mathrm{y}$ el anonimato y confidencialidad de los resultados.

Al culminar su participación, las madres recibieron una retribución económica equivalente a 15 dólares y un DVD con la observación filmada. Finalmente, las madres tuvieron la posibilidad de tener una sesión de orientación psicológica con dos miembros del equipo de investigación en temas de crianza de sus hijos. Cabe mencionar que, de ser necesario, el equipo de investigación contaba con un directorio de profesionales a los cuales derivar aquellos casos que podrían estar en riesgo.

\section{Instrumentos}

Massie-Campbell Attachment During Stress Scale (ADS).

La ADS es una guía de observación diseñada para la evaluación de la interacción bebé-cuidador en situaciones de estrés moderado -como una visita médica o episodios de separación- (Massie \& Campbell, 1983). Este instrumento valora tanto las conductas del niño como las de la madre o cuidador por separado, y para cada uno de ellos se evalúa la intensidad y frecuencia de seis conductas: mirada, vocalización, sostén, tacto, afecto y búsqueda de proximidad. La calificación de cada conducta se realiza en un continuo de cinco niveles, que van desde su ausencia hasta una fuerte manifestación, y a partir de ellas se obtiene una clasificación de la interacción de la diada.

Adicionalmente, dado que el manual original no plantea un procedimiento para el cálculo de un puntaje único, Cárcamo et al. (2014) adaptaron uno en el que la presencia de puntajes de uno o dos en más de la mitad de los ítems es un indicador de apego inseguro evitativo, mientras que la presencia de puntajes de tres y cuatro en más de la mitad de los ítems es un indicador de apego seguro, y un puntaje de cinco en más de la mitad de los ítems es un indicador de apego ambivalente.

Para este estudio, la evaluación de la interacción entre la madre y su hijo con la ADS se realizó a partir de la observación de videos durante un procedimiento estructurado llevado a cabo en un laboratorio como parte de un estudio anterior. Específicamente, el procedimiento consistió en cinco momentos diferenciados: (a) se inició con un primer momento de cinco minutos de juego libre de la madre con su hijo con los juguetes colocados en el espacio; (b) seguido de un momento en el que la madre se retira de la habitación, dejando al niño solo con los juguetes durante dos minutos, aproximadamente; (c) un tercer momento, en el que la madre retorna a la habitación e interactúa con el niño durante dos minutos más; (d) un cuarto momento, en el que ingresa una persona extraña que la diada no había visto previamente y que retira todos los juguetes del espacio -a excepción de uno con el que no hubieran jugado-; y (e) un último momento, en el que la diada continúa interactuando durante un periodo de dos minutos más. El procedimiento tenía una duración aproximada de 11 minutos.

Para la calificación de la conducta observada con la ADS se utilizó el sistema propuesto por Cárcamo et al. (2014): en primer lugar, se puntuó cada una de las conductas -en una escala de 1, 2, 3, 4, 5 o no observado-; luego, cada conducta se clasificó como segura o no segura, de acuerdo con el procedimiento establecido -las conductas con puntajes de tres o cuatro se clasificaban como seguras, mientras que las que tenían puntajes de uno, dos o cinco se clasificaban como no seguras-; y, finalmente, se clasificó a las diadas como seguras cuando el $50 \%$ o más de sus conductas eran seguras, mientras que el resto fueron consideradas no seguras.

Es importante mencionar que las calificaciones de los videos fueron realizadas por un grupo de cinco codificadores expertos en el tema del apego -quienes recibieron un entrenamiento en el uso del instrumento por parte de un experto y calificaron 16 videos de entrenamiento-; y que estos, utilizando el coeficiente Kappa ajustado a la prevalencia y al sesgo (PABAK, por sus siglas en inglés), 
alcanzaron una confiabilidad inter-observador moderada de .56. Finalmente, para asegurar la confiabilidad de las puntuaciones en este estudio, el $30 \%$ de los videos fueron calificados por dos observadores independientes, y se encontró que la confiabilidad inter-evaluador PABAK fue de moderada (.45) para la clasificación global de las diadas, y adecuada, o fair $(M=.36$; $\operatorname{Min}=.09, \operatorname{Max}=.64)$ para la clasificación por conducta.

\section{Attachment Q-Set (AQS) versión 3.0.}

El AQS, de Waters (1995), es un instrumento de observación que, mediante la metodología Q Sort, evalúa la organización de la conducta de base segura con la finalidad de caracterizar la seguridad del apego. Específicamente, se utilizó la adaptación lingüística realizada por Nóblega (2012), de la versión en español (Posada et al., 1999), que consta de 90 ítems que describen diferentes conductas del niño. Tal como se detalló en la introducción, este instrumento tiene adecuadas características psicométricas para la evaluación de los niños de 12 a 69 meses (Cadman et al., 2017; Nóblega, 2012; Nóblega et al., 2016; Posada et al., 2013; Posada et al., 2016; Solomon \& George, 2016; Vaughn, et al., 2007); y fue utilizado previamente en el contexto peruano con niños menores de 12 meses (Nóblega et al., 2016).

Para la investigación de la cual se deriva este estudio, se filmó la interacción libre de la madre con su hijo durante aproximadamente una hora, y el video fue calificado por dos evaluadores independientes. Con el objetivo de mejorar la calidad de las puntuaciones obtenidas de la conducta materna, la calificación de las observaciones se realizó en dos etapas, siguiendo los procedimientos realizados en estudios previos (Bárrig, 2004; Nóblega, 2012; Posada, Carbonell, Alzate \& Plata, 2004; Posada et al., 1995; Posada et al., 2002; Vaughn et al., 2007).

En este estudio, la confiabilidad inter-evaluador promedio de la escala en la primera etapa fue buena, de $.83(D E=0.07$, Min $=.65$ y Max = .92), mientras que, en la segunda etapa, para cada caso se identificaron los ítems en los que había alta discrepancia entre las calificaciones dadas por ambos observadores (diferencia de más de tres puntos). Estos ítems fueron discutidos a partir de referentes comportamentales observados durante la visita, luego se llegó a una descripción compartida del comportamiento del niño. De esta manera, las puntuaciones estuvieron constituidas por el promedio de las puntuaciones dadas por ambos observadores a cada ítem.

De acuerdo con el procedimiento propuesto para el instrumento, la seguridad del apego de cada niño se calcula a partir de la correlación de los puntajes otorgados por el observador a cada uno de los 90 ítems y las puntuaciones que conforman un criterio consensuado por expertos respecto a los puntajes de un niño idealmente seguro (Cadman et al., 2017). Esta puntuación teórica se denomina criterio de seguridad y ha sido validada en diferentes contextos, incluido el peruano (Cassibba, van IJzendoorn \& D'Odorico, 2000; Nóblega, 2012; Posada et al., 1995).

Cabe mencionar que en la propuesta del AQS no existe un punto de corte que determine una clasificación de apego a partir de los puntajes obtenidos (van IJzendoorn et al., 2004); no obstante, en el estudio realizado por Van Bakel y Riksen-Walraven (2004) con 129 diadas holandesas se encontró que la media de la seguridad de apego de los niños clasificados como seguros fue significativamente mayor a la de los niños clasificados como inseguros, a partir de la evaluación con el SSP $\left(M_{\text {niños seguros }}=.32, D E_{\text {niños seguros }}\right.$ $=.25, M_{\text {niños inseguros }}=.12, D E_{\text {niños inseguros }}=.24 ; t=4.34, p$ $<.01$ ). De la misma manera, van IJzendoorn et al. (2004) reportaron que la media de los puntajes de seguridad de la población normal difería significativamente de la media de la población clínica $\left(M_{\text {población tipica }}=.32, D E_{\text {población típica }}\right.$ $=.16 ; M_{\text {población clinica }}=.21, D E_{\text {población clinica }}=.08 ; t(297.20)$ $=16.06, p<.01)$. Y, de igual forma, Geenen y Conveleyn (2014) hacen una diferenciación entre el apego seguro e inseguro a partir del puntaje de .32 en el AQS. Con base en estas evidencias, se puede considerar como seguras aquellas diadas con puntuaciones AQS por encima de .32.

Q-Sort del Comportamiento Materno (MBQS) versión 2.1.

El MBQS, de Pederson y Morán (1995), es un instrumento de observación que evalúa la sensibilidad materna por medio de la metodología Q Sort para describir la conducta de la madre, a partir de 90 ítems. Este instrumento ha sido validado en diversos contextos (Posada et al., 2013; Posada et al., 2016), y para esta investigación se utilizó la versión adaptada por Posada et al. (2002) al contexto latinoamericano.

Las observaciones de la sensibilidad materna se realizaron de manera simultánea a las de la conducta del niño del AQS. Con el objetivo de mejorar la calidad de las puntuaciones obtenidas de la conducta materna se realizó el mismo procedimiento en dos etapas (descrito para el AQS). Las codificadoras fueron dos personas independientes entre sí y de las codificadoras del AQS. En la primera etapa, las calificaciones de ambas observadoras se correlacionaron y se obtuvo un promedio de confiabilidad inter-observador bueno, de $82(D E=0.09)$. En general, para 31 de los casos, el mínimo obtenido fue de .61 y el máximo de .92 , y en el caso restante se obtuvo una confiabilidad moderada, de.59, por lo que el video de la interacción fue codificado por una 
tercera evaluadora. Con este procedimiento, la confiabilidad inter-observador de este video ascendió a .75 .

Finalmente, de acuerdo con el procedimiento preestablecido para el instrumento, se obtuvo un puntaje global de sensibilidad materna para cada madre a partir de la correlación entre sus puntaciones a cada ítem y el criterio de sensibilidad materna establecido a partir del juicio de expertos (Pederson \& Moran, 1995).

\section{Procedimiento}

El presente es un estudio cuantitativo transeccional que se deriva de un proyecto de investigación de mayor amplitud (ATI [Uruguay] \& Pontificia Universidad Javeriana de Colombia) realizado en diversos contextos, en la cual se explora la relación entre los precursores del apego y la sensibilidad materna en niños menores a un año.

En este trabajo se llevaron a cabo dos reuniones con las participantes. La primera se realizó en la casa de la madre, y en ella las participantes firmaron el consentimiento informado e informaron sobre sus datos sociodemográficos; luego, se observó y filmó la interacción de la diada durante una hora; se les solicitó jugar durante cinco minutos con su niño en un espacio fijo; y, por último, se les entregó un instrumento para evaluar sus representaciones de apego. La segunda reunión se realizó en una cámara Gesell, y en esta las madres siguieron un procedimiento estructurado de separaciones y reuniones -actividad que también fue filmada-; al finalizar el procedimiento, se recolectó información sobre sus prácticas de cuidado y el uso de la canción de cuna durante la crianza. Finalmente, se realizó la sesión de orientación psicológica antes descrita.

Para este estudio se utilizaron, por un lado, las calificaciones de la seguridad del apego y de la sensibilidad materna (AQS y MBQS), obtenidas a partir de la observación de la interacción entre madre y niño en casa; $y$, por otro, las filmaciones de la interacción de las mismas diadas en el contexto de laboratorio, las cuales fueron calificadas mediante el ADS.

\section{Análisis de datos}

Para realizar los análisis estadísticos se utilizó el paquete de datos IBM SPSS Statistics 22, y, siguiendo las recomendaciones dadas por Cárcamo et al. (2014), se decidió utilizar solo la escala de infante para todos los análisis.

En primer lugar, se realizaron los análisis correspondientes para identificar la normalidad de la distribución de las puntuaciones y la homogeneidad de varianza. A partir del análisis de los indicadores de asimetría y la curtosis, así como de la prueba Shapiro-Wilk y los gráficos de normalidad, se concluyó que los puntajes continuos de seguridad del AQS eran de $S W(32)=.949$, Curtosis $=-.094$ y Asimetría $=-.675$; mientras que para la sensibilidad materna eran de $S W(32)=.905$, Curtosis $=.125$ y Asimetría $=-.880$.

Asimismo, a nivel específico y partiendo de los mismos indicadores, se concluyó que las puntuaciones de seguridad en los grupos de niños seguros y no seguros contaban con distribuciones normales: $S W(25)$ seg, grupo niños seguros $=.938$, Curtosis $_{\text {seg, grupo niños seguros }}=1.018$ y Asimetría ${ }_{\text {seg, grupo niños seguros }}=$ -.975) para los seguros; versus $S W(7)_{\text {seg. grupo de niños no seguros }}=$ .913 , Curtosis ${ }_{\text {seg, grupo niños no seguros }}=-1.189$ y Asimetría niños no seguros $=-.269$ para los inseguros. Al igual que para las puntuaciones de la sensibilidad de las madres de los niños seguros formados a partir de las clasificaciones dadas por el ADS: $S W(25)_{\text {sen. grupo niños seguros }}=.873$, Curtosis ${ }_{\text {sen. grupo niños }}$ seguros $=.122$ y Asimetría ${ }_{\text {sen. grupo niños seguros }}=-1.010$ ) para los seguros; y $S W(7)_{\text {sen. grupo niños no seguros }}^{=} .939$, Curtosis sen. grupo niños no seguros $=1.997$ y Asimetría ${ }_{\text {sen. grupo niños no seguros }}=-.007$ para los inseguros.

Luego, para tener una evidencia de validez convergente de la ADS, se analizó la relación entre la clasificación de apego seguro y no seguro del ADS con los puntajes de seguridad del AQS, utilizando la correlación biserial puntual. Asimismo, se realizó la diferencia de medias en las puntuaciones del AQS entre los niños clasificados con apego seguro e inseguro con la ADS a partir del estadístico t de Student. A continuación, se utilizaron los estadísticos Chi Cuadrado y V de Cramer para examinar la concordancia entre las clasificaciones seguro vs. no seguro de la ADS y del AQS; y, para realizar una clasificación de apego seguro vs. no seguro con las puntuaciones en el AQS, se utilizó .32 como punto de corte; esto porque en estudios previos se reporta este puntaje como la media de seguridad en diferentes grupos poblacionales no clínicos (Geenen \& Conveleyn, 2014; van Bakel \& Riksen-Walraven, 2004; van IJzendoorn et al., 2004).

Por potra parte, para evidenciar la validez divergente de la escala se analizó la relación entre los puntajes del ADS y del MBQS (sensibilidad materna), y se utilizó el contraste de medias mediante el estadístico t de Student para comparar los puntajes de sensibilidad materna en el grupo de niños seguros y en el grupo de no seguros.

Posteriormente, a partir de los resultados del análisis de discrepancias, se procedió a analizar la sensibilidad, especificidad, valor predictivo positivo y valor predictivo negativo de la escala ADS, utilizando las clasificaciones AQS como criterio de referencia.

Finalmente, se realizó un análisis de Característica Operativa del Receptor (COR) para identificar una forma de clasificar a los niños con apego seguro que permita optimizar la sensibilidad y especificidad de la ADS, para lo cual se calculó una puntuación continua para la ADS sumando las puntuaciones de los siete ítems convertidas a la clasificación 
136

de seguro (1 punto) o no seguro (0 puntos). Se utilizó el índice de Youden para definir un punto de corte óptimo.

\section{Resultados}

A continuación se presenta, en primer lugar, los estadísticos descriptivos de las puntuaciones obtenidas por el grupo en cada uno de los instrumentos; después, se muestran los resultados de los análisis realizados para evaluar las evidencias de validez convergente de la escala, utilizando los puntajes y clasificaciones obtenidas a partir del AQS; en tercer lugar, se presentan los resultados encontrados para el análisis de las evidencias de la validez divergente de la escala, utilizando los puntajes de la sensibilidad materna (MBQS); y, finalmente, para evidenciar la validez de criterio, se exponen los resultados de los análisis de sensibilidad, especificidad y valores predictivos de la escala, así como de los análisis de Curvas ROC, realizados para encontrar un nuevo punto de corte más apropiado.

\section{Estadísticos descriptivos de los puntajes}

La seguridad del apego evaluada a partir del AQS obtuvo una media de $.195(D E=.235 ;$ Min $=-.358 ;$ Max $=.586)$. A partir de la clasificación dicotómica del ADS se identificó que un $78.1 \%$ de los niños participantes pertenecía al grupo de apego seguro y un $21.9 \%$ al grupo no seguro. Asimismo, se encontró una media de $.543(D E=.242 ; \operatorname{Min}=-.021$; Max $=.834$ ) de sensibilidad materna, según los resultados del MBQS.

Evidencia de validez convergente: clasificaciones de AQS y ADS.

Considerando la clasificación dicotómica (apego seguro vs. apego no seguro) de la ADS, se encontró que una relación positiva entre esta y las puntuaciones del AQS $\left(r_{\mathrm{bp}}=.408\right.$, $p=.021)$, lo cual quiere decir que, a mayor seguridad del apego evaluada con el AQS, los niños tienden a ser clasificados también como seguros a partir de sus puntuaciones en la ADS.

Asimismo, a partir de la puntuación en la ADS, se clasificó a los niños en dos grupos (apego seguro o apego no seguro) y se llevó a cabo una comparación de medias de la seguridad del apego para saber si existían diferencias. Los resultados mostraron que los niños clasificados como no seguros tenían puntuaciones significativamente menores de seguridad en el AQS $\left(M_{\text {niños seguros }}=.25, D E_{\text {niños seguros }}=.22\right.$, $M_{\text {niños no seguros }}=.02, D E_{\text {niños no seguros }}=.22, t(30)=-2.446$, $p=.02, d=.89 ; 95 \%$ IC $=[-.419 ;-.037])$.

De la misma manera, la relación entre las clasificaciones dicotómicas de apego seguro y no seguro dadas por la ADS y el AQS es significativa $\left[\chi^{2}(1, N=32)=4.69, p=.03\right.$, $V=.383]$; y, de manera general, como se observa en la Tabla 1, el $56.3 \%$ de los participantes (18 niños) obtuvo la misma clasificación en ambos instrumentos; todos los participantes clasificados como seguros en el AQS obtuvieron la misma clasificación en la ADS; y todos los participantes clasificados como no seguros a partir de la ADS obtuvieron puntuaciones compatibles con esta categorización en el AQS. No obstante, cabe resaltar que el $56 \%$ del total de participantes (14 niños) clasificados como seguros por la ADS fueron clasificados como inseguros según el AQS.

Tabla 1.

Tabla cruzada Clasificación ADS y Clasificación AQS seguro - inseguro $(N=32)$

\begin{tabular}{cccc}
\hline \multirow{2}{*}{ ADS } & \multicolumn{2}{c}{ AQS } & \multirow{2}{*}{ Total } \\
\cline { 2 - 3 } & Inseguro & Seguro & \\
\hline Inseguro & 7 & 0 & $7(21.9 \%)$ \\
Seguro & 14 & 11 & $25(78.1 \%)$ \\
Total & $21(65.6 \%)$ & $11(34.4 \%)$ & $32(100 \%)$ \\
\hline
\end{tabular}

Evidencias de validez divergente: comparación de la sensibilidad de las madres de los niños clasificados con apego seguro y las madres de niños clasificados con apego inseguro

Las madres del grupo de participantes clasificados en la ADS con apego seguro no obtuvieron puntuaciones significativamente mayores en sensibilidad materna que las madres de los niños clasificados como inseguros $(M$ madres de niños seguros $=.55, D E$ madres de niños seguros $=.26: M_{\text {madres de }}$ niños no seguros $=.52, D E \underset{\text { madres de niños no seguros }}{=} .19 ; t(30)=-.34$, $p=.74, d=-.123 ; 95 \% I C=[-.249 ; .179])$.

Sensibilidad, especificidad y valores predictivos del ADS.

La escala ADS obtuvo una sensibilidad de $33.3 \%$, con un $I C 95 \%=$ [15.48; 56.90], es decir, que, al utilizar la clasificación de apego seguro o no seguro a partir de la ADS, hay entre 15 y $57 \%$ de probabilidad de identificar correctamente los casos que según el AQS tienen un apego no seguro. Asimismo, la ADS tiene una especificidad de $99 \%$, IC $95 \%=[67.85 ; 99.16]$, es decir, una probabilidad de 68 a $99 \%$ de identificar correctamente los casos con apego seguro.

Con respecto al valor predictivo positivo de la escala de la ADS, los resultados muestran un $99 \%$, IC $95 \%=$ [56.09; 98.68], de probabilidad de que un niño clasificado con apego inseguro a partir del AQS tenga la misma clasificación con la escala ADS. Sin embargo, respecto al valor predictivo negativo, los resultados muestran que solo hay un $44 \%$, IC $95 \%=[25.02 ; 64.7]$, de probabilidad de que 


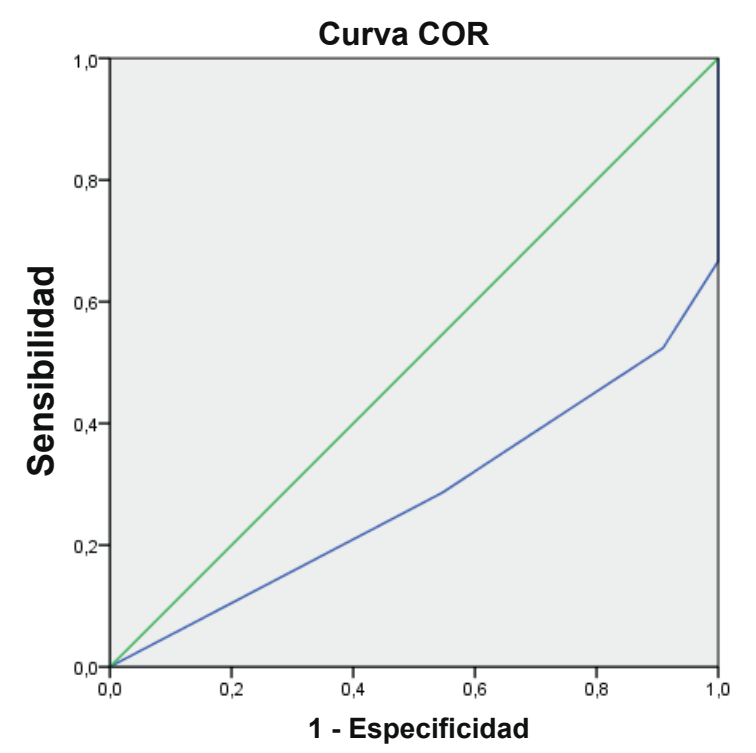

Figura 1. Curva ROC de los puntajes continuos del ADS, utilizando los puntajes AQS como criterio (punto de corte de .32 , de acuerdo con estudios previos).

un caso clasificado como un niño con apego seguro sea clasificado del mismo modo con el AQS.

A partir de los resultados anteriores se realizaron los análisis de Curva ROC y el índice de Youden con la finalidad de encontrar un punto de corte para la ADS que pueda optimizar tanto su nivel de sensibilidad como el de especificidad. Para ello, se consideró las puntuaciones del AQS (con el punto de corte de .32) como criterio y se utilizó una puntuación continua para la ADS, sumando las puntuaciones de los ítems.

En la Figura 1 se observa la curva ROC. Acá, el indicador de la capacidad discriminativa de la ADS (el área debajo de la curva) corresponde a .28 (IC $95 \%=[.105 ; .453]$; $\mathrm{z}=.09, \mathrm{p}=.04)$, lo que indicaría que el criterio propuesto no es mejor que el azar.

A través del índice de Youden, se define que el punto de corte óptimo para considerar un niño con apego seguro a partir de la ADS sería tener 4.5 conductas clasificadas como seguras (véase Tabla 2).

Tabla 2.

Sensibilidad, especificidad e índices de Youden para los diferentes puntos de corte obtenidos a partir del análisis de Curvas ROC

\begin{tabular}{cccc}
\hline $\begin{array}{c}\text { Punto de corte } \\
\text { evaluado }\end{array}$ & Sensibilidad & 1 - Especificidad & $\begin{array}{c}\text { Índice de } \\
\text { Youden }\end{array}$ \\
\hline 2.5 & 0.857 & 1 & 0.857 \\
3.5 & 0.667 & 1 & 0.667 \\
4.5 & 0.524 & 0.909 & 0.433 \\
5.5 & 0.286 & 0.545 & -0.169 \\
6.5 & 0.095 & 0.182 & -0.723 \\
\hline
\end{tabular}

Dado que las conductas constituyen unidades indivisibles, este dato se redondeó y se estableció como nuevo punto de corte el tener cinco o más conductas clasificadas como seguras. Esta nueva forma de clasificación brindó una sensibilidad de $47.6 \%($ IC $95 \%=[26.4 ; 69.7])$ y una especificidad de $90.9 \%$ (IC $95 \%=[57.1 ; 99.5])$.

De este modo, como se observa en la Tabla 3, al utilizarse cinco o más conductas seguras en el ADS para considerar a un niño con apego seguro a partir de la escala, se obtuvo un $65.6 \%$ de niños clasificados con apego seguro; y la correlación con la seguridad del apego medida por el AQS mejoró ligeramente $\left(r_{\mathrm{bp}}=.43, p=.01\right)$. Asimismo, la asociación entre ambas clasificaciones se mantuvo significativa $\left[\chi_{(1, N=32)}^{2}=4.75, p=.03, V=.38\right]$. Con respecto a la concordancia entre las clasificaciones de apego seguro y no seguro dadas por ambos instrumentos, se encontró un acuerdo en el $62.5 \%$ de los casos (20).

Tabla 3

Tabla cruzada Clasificación ADS con nuevo punto de corte y Clasificación AQS seguro - inseguro con punto de corte $.32(n=32)$

\begin{tabular}{cccc}
\hline \multirow{2}{*}{ ADS } & \multicolumn{2}{c}{ AQS } & \multirow{2}{*}{ Total } \\
\cline { 2 - 3 } & Inseguro & Seguro & \\
\hline Inseguro & 10 & 1 & $7(34.4 \%)$ \\
Seguro & 11 & 10 & $25(65.6 \%)$ \\
Total & $21(65.6 \%)$ & $11(34.4 \%)$ & $32(100 \%)$ \\
\hline
\end{tabular}

\section{Discusión}

El presente estudio tuvo como objetivo generar evidencias de validez convergente y discriminante de la ADS, así como de validez de criterio. Para ello, se utilizaron los datos generados en un estudio previo con un grupo de 32 madres y de sus hijos de 8 a 10 meses de edad.

En primer lugar, la ADS en su modalidad de clasificación dicotómica (seguro vs. no seguro), obtuvo adecuadas evidencias de validez convergente, expresadas en su relación positiva y moderada con las puntuaciones de seguridad dadas por el AQS, y en la mayor puntuación en el AQS obtenida por los niños clasificados como seguros respecto a los clasificados como no seguros en la ADS. Además, es de resaltar que la magnitud de la relación hallada en este estudio es similar a la relación entre los puntajes del AQS y la clasificación dada por el SSP reportada por van IJzendoorn et al. (2004). Estos resultados respaldarían el uso de la ADS en el campo de la investigación del apego de los niños menores a 18 meses, considerando que el AQS es el segundo instrumento más utilizado en las investigaciones sobre apego en diversos contextos (van IJzendoorn et al., 2004), incluido el latinoamericano. 
Sin embargo, no se pudo hallar una adecuada evidencia de validez divergente con los puntajes del MBQS (Pederson $\&$ Moran, 1995), dado que no se encontraron diferencias significativas en la sensibilidad de las madres de los niños clasificados como seguros y no seguros por la ADS. Estos resultados son similares a los encontrados por Cárcamo et al. (2014) -quienes utilizaron la Escala de Sensibilidad, de Ainsworth, Bell y Stayton (1974)-, lo cual indicaría que el apego de los niños medido a través de la ADS no se encuentra relacionado con la conducta de sus madres. Una posible explicación para esto podría ser la diferencia entre los contextos de evaluación de cada instrumento: el apego evaluado por la ADS en esta investigación fue medido en una situación de estrés de aproximadamente 15 minutos, mientras que la sensibilidad de la madre fue evaluada en una situación de interacción cotidiana de aproximadamente una hora. Esta explicación requiere ser profundizada en estudios posteriores donde se controlen diversas variables metodológicas que podrían estar interfiriendo en los resultados obtenidos.

Por otro lado, se analizó la concordancia entre las clasificaciones dadas por la ADS y el AQS, considerando para este último una clasificación categórica de apego seguro no seguro, y los resultados mostraron que ambas medidas difieren significativamente en las clasificaciones de seguridad del apego de las diadas. Específicamente, se observó que la ADS muestra una tendencia a la sobrerrepresentación del apego seguro, y los análisis de sensibilidad, especificidad, valores predictivos positivos y valores predictivos negativos reforzaron este aspecto al mostrar que la ADS tendría dificultades para identificar correctamente los casos de apego no seguro. Estos resultados coinciden con un estudio previo que utilizó el SSP como criterio (Cárcamo et al., 2014); no obstante, la diferencia es que en dicho estudio se consideró una clasificación tricotómica para los resultados de la ADS (apego seguro, inseguro ambivalente e inseguro evitativo).

Además de esto, los resultados de los análisis de curvas ROC permitieron proponer una nueva forma de clasificar a un niño con apego seguro a partir de sus puntuaciones en la ADS. Así, el análisis sugiere clasificar el apego seguro cuando el niño obtenga cinco de las siete conductas clasificadas como seguras. Esta forma de clasificación incrementó la sensibilidad de la escala de $33 \%$ a $47.6 \%$, incrementó ligeramente la relación con las puntuaciones del AQS y disminuyó la frecuencia de la clasificación del apego seguro, colocándola en un valor más acorde al reportado por la literatura internacional; aunque la especificidad de la escala disminuyó de $99 \%$ a $90.9 \%$. En conjunto, estos resultados mostrarían que, incluso ajustando la forma de clasificar a un niño con apego seguro, la ADS no presentaría suficientes evidencias que avalen su uso como prueba de tamizaje para el descarte de un apego de tipo no seguro.

Es importante recalcar que aspectos como el tamaño de la muestra del estudio, así como las propias propiedades psicométricas del criterio, cobran especial relevancia para los resultados de una validez de criterio (Evers et al., 2013). En ese sentido, si bien en este estudio se contó con un criterio de referencia ampliamente avalado por la literatura, el tamaño pequeño de la muestra supondría una limitación para la exactitud de sus resultados. Por ello, se recomienda reproducir estos procedimientos con una muestra más grande y representativa.

Asimismo, el hecho de que los resultados de ambas medidas estén relacionados y al mismo tiempo las clasificaciones obtenidas a partir de ambos instrumentos difieran podría denotar diferencias en la conceptualización del apego en la ADS y el AQS, y esto llevaría a la necesidad de que en futuros estudios se revise y ajuste el contenido de los ítems de la ADS para que sea más compatible con otros instrumentos, como el AQS. En esta misma línea, futuras investigaciones podrían identificar las conductas propuestas en la ADS que contribuyen más a la sobrerrepresentación del apego seguro y realizar modificaciones sobre los mismos.

Por último, la literatura centrada en la evaluación psicométrica de los instrumentos psicológicos sostiene que, si bien todas las evidencias de validez son importantes para la valoración de los tests, las evidencias de validez de criterio son especialmente importantes cuando se pretende usar las medidas para realizar predicciones (Evers et al., 2013). En ese sentido, estudios como el presente cobran relevancia dada la reciente extensión del uso de la ADS como prueba de tamizaje en evaluaciones poblacionales. $\mathrm{Y}$, en este sentido, a pesar de las limitaciones descritas, el presente estudio forma parte de los esfuerzos por generar evidencias psicométricas de la ADS y es el primero en evaluar su sensibilidad, especificidad y valores predictivos. Se recomienda seguir estudiando estas características de la escala por la importancia que tiene el instrumento para las evaluaciones poblacionales y la investigación de un tema tan complejo y de creciente interés como lo es el del apego.

\section{Referencias}

Ainsworth, M. D. S., Bell, S. M., \& Stayton, D. J. (1974). Infant-mother attachment and social development: Socialization as a product of reciprocal responsiveness to signals. En M. P. M. Richards (Ed.), The integration of a child into a social world (pp. 99-135). Londres: Cambridge University Press. 
Ainsworth, M., Blehar, M., Waters, E., \& Wall, S. (1978). Patterns of attachment. A psychological study of the Strange Situation. Nueva Jersey: Lawrence Erlbaum Associates.

Bárrig, P. (2004). Relation between children's attachment representations and secure base behavior (Tesis de maestría). Purdue University, Indiana, Estados Unidos de América.

Bowlby, J. (1969/1982). Attachment and loss: Attachment. Nueva York: Basic Books.

Bowlby, J. (1988). A secure base. Nueva York: Basic Books.

Buitrón, V. (2011). Apego en hijos de madres adolescentes víctimas de abuso sexual en Lima Metropolitana (Tesis de licenciatura). Pontificia Universidad Católica del Perú, Lima, Perú. Recuperado de http://tesis.pucp.edu. pe/repositorio/ handle/123456789/625

Cárcamo, R., Van IJzendoorn, M., Vermeer, H., \& Van der Veer, R. (2014). The validity of the Massie-Campbell Attachment During Stress Scale (ADS). Journal of Child and Family Studies, 23(5), 767-775. doi:10.1007/s10826-013-9728-z

Cassibba, R., Van IJzendoorn, M. H., \& D’Odorico, L. (2000). Attachment and play in child centers: Reliability and validity of the attachment Q sort for mother and professional caregiver in Italy. International Journal Behavioral Development, 24(2), 241-255. doi:10.1080/016502500383377.

Cassidy (2016). The nature of the child's ties. En J. Cassidy \& P.R. Shaver (Eds.), Handbook of attachment: Theory, research, and clinical applications (3. ${ }^{\mathrm{a}}$ ed.) (pp. 3-24). Nueva York: Guilford Press.

Cadman, T., Diamond, P. R., \& Fearon, P. (2017). Reassessing the validity of the attachment Q-sort: An updated metaanalysis. Infant and Child Development. Advance online publication. doi:10.1002/icd.2034

Clarke-Stewart, K., Goossens, F., \& Allhusen, V. (2001). Measuring infant-mother attachment: Is the Strange Situation enough? Social Development, 10(2), 143-169. doi:10.1111/1467-9507.00156

Conde, G., Nóblega, M., Fourment, K., \& Nuñez del Prado, J. (2015, Agosto). Secure Base Behavior in preschoolers: $A Q S$ comparison between home and park observations in Lima, Perú. Poster presentado en la International Attachment Conference - IAC, Nueva York, NY.

Dávila, D. (2013). Apego y sensitividad materna en madres y niños preescolares del distrito de Los Olivos (Tesis de licenciatura). Pontificia Universidad Católica del Perú, Lima, Perú. Recuperado de http://tesis.pucp.edu.pe/repositorio/ handle/123456789/5010

Evers, A., Fernández, J. M., Hagemeister, M., Hagemeister, C., Hstmaelingen, ... Bartram, D. (2013). Assessing the quality of tests: Revision of the EFPA review model. Psicothema, 25(3), 283-291. doi:10.7334/psicothema2013.97

Evers, A., Hagemeister, C., Hostmaelingen, A., Lindley, P., Muñiz, J., \& Sjöberg, A. (2013). EFPA Review model for the description and evaluation of psychological and educational tests. Test review form and notes for reviewers. Version 4.2, 6. Recuperado de http://www.efpa.eu/professional-development/assessment

Figueroa, F., Vergara, V., \& Santelices, M.P. (2012). Enhancing early attachment: Design and pilot study of an intervention for primary health care dyads. Journal of Child Health Care, 16(17), 82-91. doi:10.1177/1367493512447533

Geenen, G., \& Corveleyn, J. (2014). Vinculos protectores: Apego en padres e hijos en vulnerabilidad. Fondo Editorial de la Pontificia Universidad Católica del Perú.

Kotliarenco, M., Gomez, E., Muñoz, M., \& Armijo, I. (2009). Evaluación pre-post del desarrollo psicomotor y el estilo de apego en usuarios de los Centros de Desarrollo Infantil Temprano. SUMMA Psicológica UST, 6(2), 89-104.

Jin, M.K., Jacobvitz, D., Hazen, N., \& Jung, S. (2012). Maternal sensitivity and infant attachment security in Korea: Cross-cultural validation of the Strange Situation. Attachment \& Human Development, 14(1), 33-44. doi:10.1080/14 616734.2012 .636656$.

Lecannelier, F., Undurraga, V., Olivares, A. M., Rodriguez, J., Nuñez, J. C., ... Larraín, C. (2009). Estudio de eficacia sobre dos intervenciones basadas en el fomento del apego temprano en díadas madre-bebé provenientes de la región Metropolitana en Santiago de Chile. Revista Argentina de Clínica Psicológica, 18(2), 143-156.

Leigh, F., Vergara, V., \& Santelices, M. (2012). Enhancing early attachment: Design and pilot study of an intervention for primary health care dyads. Journal of Child Health Care, 17(1), 82-91. doi:10.1177/1367493512447533

Massie, H., \& Campbell, K. (1983). The Massie-Campbell Scale of Mother-Infant Attachment Indicators during Stress. En J. Call, E. Galenson \& R. Tyson (Eds.), Frontiers of infant psychiatry (pp. 394-412). Nueva York: Basic Books.

Massie, H., \& Campbell, K. (1992). The Massie-Campbell Scale of mother-infant attachment indicators during stress. For use during the pediatric examination and other childcare situations. Berkeley: Instruction Guide.

Nóblega, M. (2012). Conducta de base segura y sensitividad en niños y madres del distrito de los Olivos (Tesis de doctorado). Pontificia Universidad Católica del Perú, Lima, Perú. Extraída de http://tesis.pucp.edu.pe/repositorio//handle/123456789/4491

Nóblega, M., Bárrig, P., Conde, G., Nuñez del Prado, J., Carbonell, O. A., ... Bauer, M. (2016). Cuidado materno y seguridad del apego antes del primer año de vida. Universitas Psychologica, 15(1), 245 - 260. doi: 10.11144/Javeriana. upsy15-1.cmsa

Nóblega, M., Fourment, K., Núñez del Prado, J., Conde, G., \& Bahamonde, D. (2015, Agosto). Maternal sensitivity and child attachment security at 3-5 years old: Differences between low and middle socioeconomic status at Lima, Peru. Poster presentado en el International Attachment Conference - IAC, Nueva York, NY. 
140

Pederson, D. R., \& Moran, G. (1995). Appendix B. Maternal Behavior Q-set. Monographs of the Society for Research in Child Development, 60(2-3 Serial No. 244), 247-254. doi: $10.2307 / 1166182$

Pederson, D. R., Moran, G., \& Bento, S. (1999). Maternal Behaviour Q-sort. Recuperado de http://ir.lib.uwo.ca/psycho logypub/1

Posada, G., Carbonell, O., Alzate, G., \& Plata, S. (2004). Through Colombian lenses: Ethnographic and conventional analyses of maternal care and their associations with secure base behavior. Developmental Psychology, 40(4), 508-518. doi:10.1037/0012-1649.40.4.508

Posada, G., Gao, Y., Wu, F., Posada, R., Tascon, M., ... Synnevaag, B. (1995). The secure-base phenomenon across cultures: Children's behavior, mothers' preferences, and experts' concepts. Monographs of the Society for Research in Child Development, 60(2/3), 27-48. doi:10.2307/1166169

Posada, G., Jacobs, A., Carbonell, O., Alzate, G., Bustamante, M., \& Arenas, A. (1999). Maternal care and attachment security in ordinary and emergency contexts. Developmental Psychology, 35(6), 1379-1388. doi:10.1037//00121649.35.6.1379

Posada, G., Jacobs, A., Richmond, M., Carbonell, O., Alzate, G., Bustamante, M., \& Quiceno, J. (2002). Maternal caregiving and infant security in two cultures. Developmental Psychology, 38(1), 67-78. doi:10.1037//0012-1649.38.1.67

Posada, G., Kaloustian, G., Richmond, M., \& Moreno, A. (2007). Maternal secure base support and preschoolers' secure base behavior in natural environments. Attachment \& Human Development, 9(4), 393-411. doi:10.1080/14616730701712316

Posada, G., Lu, T., Trumbell, J., Kaloustian, G., Trudel, M., ... Lay, K. L. (2013). Is the secure base phenomenon evident here, there, and anywhere? A cross-cultural study of child behavior and experts' definitions. Child Development, 84(6), 1896-1905. doi:10.1111/cdev.12084

Posada, G., Trumbell, J., Noblega, M., Plata, S., Peña, P., Carbonell, O. A., \& Lu, T. (2016). Maternal sensitivity and child secure base use in early childhood: Studies in different cultural contexts. Child Development, 87(1), 297-311.

Solomon J., \& George C. (2016). The measurement of attachment security and related constructs in infancy and early childhood. En J. Cassidy \& P.R. Shaver (Eds.), Handbook of attachment: Theory, research, and clinical applications (3. ${ }^{\mathrm{a}}$ ed.) (pp. 366-398). Nueva York: Guilford Press.

Tryphonopoulos, P. D., Letourneau, N., \& Ditommaso, E. (2014). Attachment and caregiver-infant interaction: A review of observational-assessment tools. Infant Mental Health Journal, 35(6), 642-656. doi:10.1002/imhj.21461
Ugarte, A. (2014). Conducta de base segura con el padre y representaciones de apego en niños preescolares (Tesis de licenciatura). Pontificia Universidad Católica del Perú, Lima, Perú. Extraída de http://tesis.pucp.edu.pe/repositorio/handle/123456789/5599

Unidad de Intervención Temprana (UIT) (s. f.) Escala massiecampbell de observación de indicadores de apego madrebebé en situaciones de stress (o escala de apego durante stress). Manual de administración, codificación y guía de conductas problemáticas en el apego. Concepción, Chile: Facultad de Psicología de la Universidad del Desarrollo

Van Bakel, H., \& Riksen-Walraven, J. (2004). AQS security scores: What do they represent? A study in construct validation. Infant Mental Health Journal, 25(3), 175-193. doi:10.1002/imhj.20001

Van IJzendoorn, M., Vereijken, C., Bakermans-Kranenburg, M., \& Marianne Riksen-Walraven, J. (2004). Assessing attachment security with the Attachment Q Sort: Metaanalytic evidence for the validity of the observer AQS. Child Development, 75(4), 1188-1213. doi:10.1111/j.14678624.2004.00733.x

Van IJzendoorn, M., Bakermans-Kranenburg, M., \& SagiSchwartz, A. (2006). Attachment across diverse sociocultural contexts: The limits of universality. En K. Rubin \& O. Boon (Eds.), Parenting beliefs, behaviors and parent-child relations (pp. 107-142). Nueva York: Psychology Press.

Vaughn, B., Coppola, G., Verissimo, M., Monteiro, L., Santos, A., ... Korth, B. (2007). The quality of maternal secure-base scripts predicts children's secure-base behavior at home in three sociocultural groups. International Journal of Behavioral Development, 31(1), 65-76. doi:10.1177/0165025407073574

Waters, E. (1995). Appendix A: The Attachment Q-set (version 3.0). Monographs of the Society for Research in Child Development, 60(2-3), 234-246. doi:10.1111/j.1540-5834.1995. tb00214.x

Waters, E., \& Deane, K. E. (1985). Defining and assessing individual differences in attachment relationships: Q-methodology and the organization of behavior in infancy and early childhood. Monographs of the society for research in child development, 41-65. 